\title{
Ketofol and High-Flow-Nasal-Oxygen for complex lower limb orthopaedic surgery in an anxious patient with Achondroplasia, severe sleep apnoea and a Chiari malformation.
}

K. Wilson, M. McNally, V. Athanassoglou, S. Galitzine.

Nuffield Orthopaedic Centre, Oxford University Hospitals NHS Foundation Trust, Oxford, UK

\section{The Case}

We present a case where a triad of infrequently used anaesthetic interventions - ultrasound scanning before CNA, the addition of ketamine to propofol (Ketofol) and high-flownasal-oxygenation (HVNO) helped to ensure safe and effective sedation for prolonged surgery.

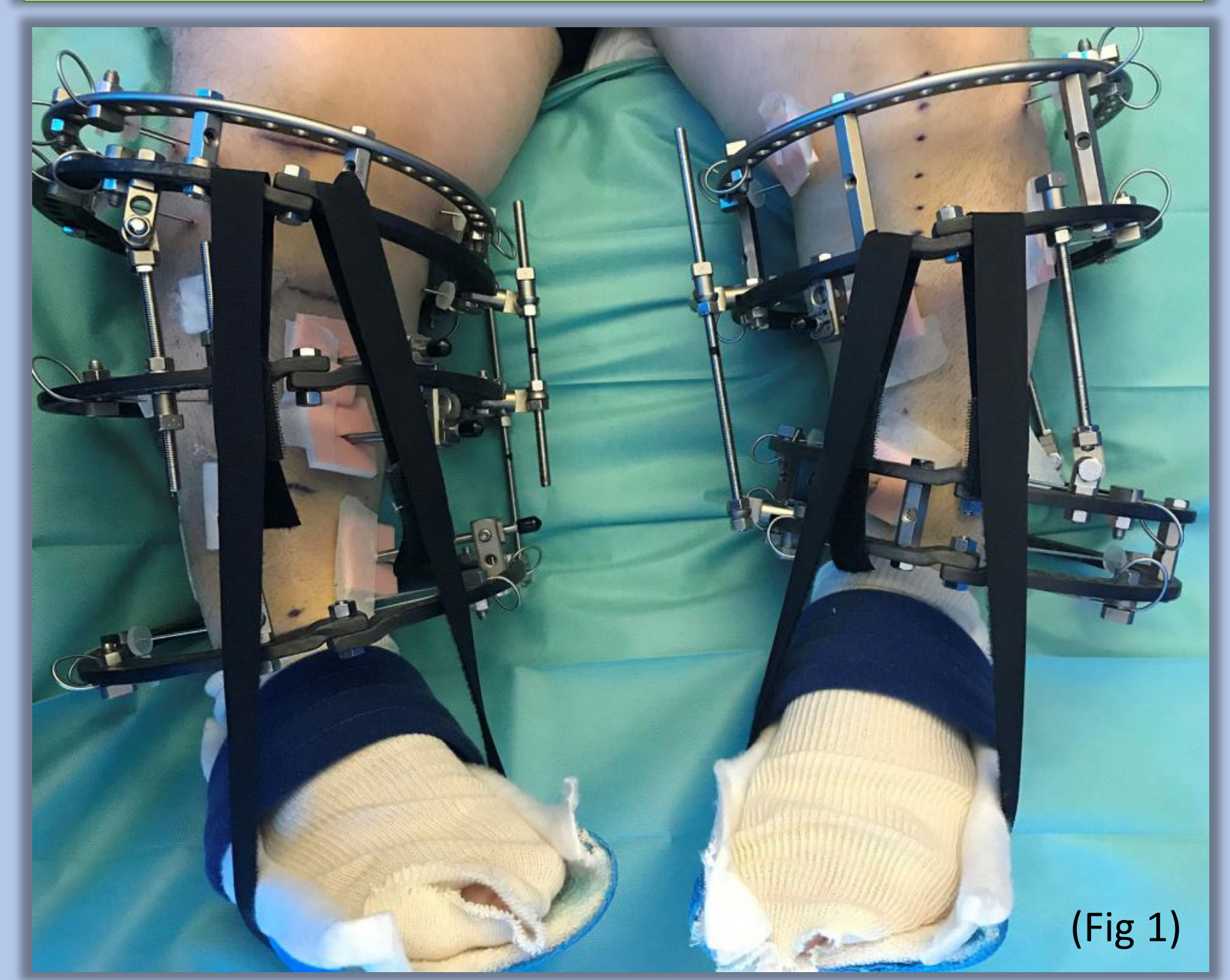

A 23-year-old man with achondroplasia required bilateral corrective Ilizarov frames (Fig 1) with osteotomies, prolonged complex surgery causing severe postoperative pain. He had typical appearance with anticipated difficult intubation, BMI 39, severe sleep apnoea, Type 1 Chiari malformation (CM-1) and significant anxiety.

The patient understood the risks of GA and consented to CNA but insisted on deep sedation. Ultrasound scanning revealed that the intercristal line was unreliable as a landmark (Fig 2) and assisted an uncomplicated placement of a lower lumbar combined spinal-epidural (CSE). $2.25 \mathrm{ml}$ of intrathecal $0.5 \%$ heavy Marcaine provided predictable and effective anaesthesia.

Deep sedation with Propofol $\mathrm{TCl}$ and increments of Midazolam caused respiratory acidosis: $\mathrm{pH} 7.25, \mathrm{pCO}_{2} 8.7 \mathrm{kPa}$. Adding Ketamine allowed a reduced $\mathrm{TCl}$ rate with effective anxiolysis, preserved airway patency, better respiratory drive and reversed acidosis: $\mathrm{pH} 7.46$ and pCO2 5.7kPa. HFNO at $30-50 \mathrm{l} / \mathrm{min}$ maintained adequate oxygenation throughout the $>5 \mathrm{hrs}$ procedure. Postoperative period was uncomplicated. Patient controlled epidural analgesia provided pain relief for 3 days postoperatively, allowing the patient to undergo physiotherapy and Ilizarov frame adjustments (Fig 2).

The patient ranked his anxiety preoperatively at $10 / 10$, intraoperatively at $0 / 10$ and postoperatively at $1 / 10$. His anaesthetic experience was

"better", "more relaxed" and "much more positive" than his previous GA.

\section{Conclusions}

For achondropastic patients requiring lower limb surgery, we recommend ultrasound assistance to secure uncomplicated neuraxial anaesthesia, and use of Ketofol with High flow nasal oxygenation to enable safe sedation and excellent patient satisfaction

\section{Discussion and Learning points}

\section{1) Anaesthesia for Achondroplasia: GA vs $\mathrm{CNA}^{1}$}

- Patients are often difficult to intubate due to a small larynx, stiff TMJ and pectus carinatum. Hyperextension of the neck should be avoided due to the high incidence of foramen magnum stenosis.

- Ventilation may impaired secondary to obstructive or central sleep apnoea and restrictive chest wall defects.

- Neuraxial blocks can be technically difficult due to kyphoscoliosis; singleinjection techniques provide an unpredictable level of block due to a narrowed epidural space and spinal canal stenosis.

\section{2) Ultrasound assisted neuraxial anaesthesia}

Type 1 Chiari malformation (CM-1) is associated with syringomyelia, kyphoscoliosis and spina bifida, while Achondroplasia is associated with kyphoscoliosis, spinal stenosis and epidural space narrowing. ${ }^{1}$

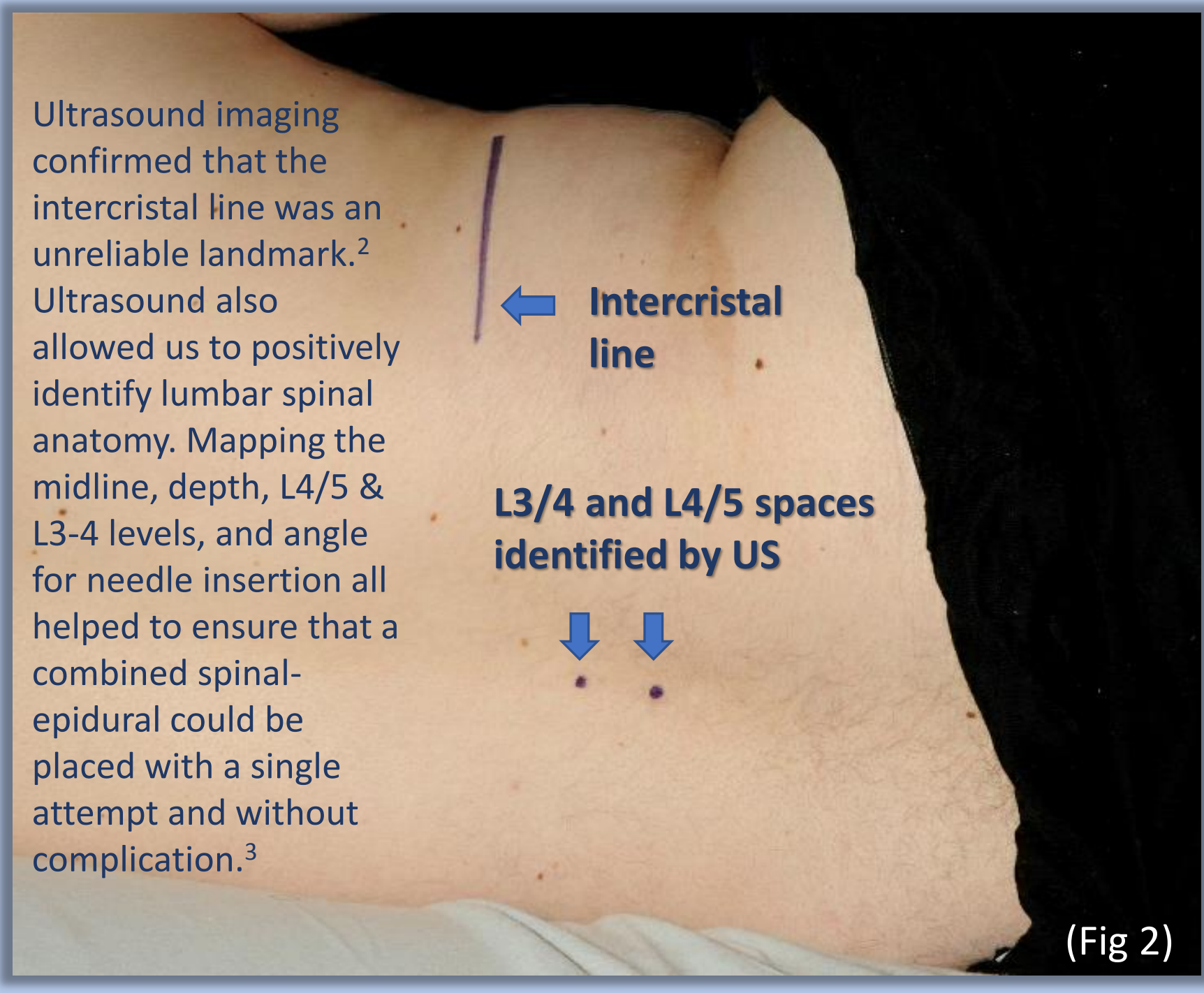

\section{3) Sedation \& sleep apnoea: "Ketofol" \& HFNO}

- The chosen sedative technique must alleviate anxiety while minimising the risks of hypoxia and hypoventilation.

- Intraoperative conversion to GA would be particularly undesirable.

- Sedation with Propofol TCI was inadequate: too little provoked anxiety, too much caused airway obstruction and respiratory acidosis.

- Adding Ketamine to Propofol (1:5 mix) successfully allowed adequate sedation while preserving airway patency and respiratory drive. ${ }^{4}$

- We believe that High Flow Nasal Oxygen (HFNO) also helped to improved the safety of procedural sedation in this patient.

- HFNO provides a baseline level of PEEP which may help to maintain an open airway and improve oxygenation. Humidification and soft nasal specs improve patient comfort and the high flow can improve ventilation and $\mathrm{CO} 2$ clearance $^{5}$.

Allman KG, Wilson IH. Oxford handbook of anaesthesia. 2016 pg193, 278, 280, 401

Margarido CB, Mikhael R, Arzola C, Balki M, Carvalho JC. The intercristal line det

anatomical landmark for neuraxial anesthesia.Can J Anaesth. 2011; 58(3):262-6.

Ghosh SM, Madjdpour C, Chin KJ. Ultrasound-guided lumbar central neuraxial block. BJA Education. 2006; 16 (7), 213-220. Rapeport DA, Martyr JW, Wang LP. The use of "ketofol" (ketamine-propofol admixture) infusion in conjunction with regional anaesthesia. Anaesth Intensive Care. 2009 Jan; 37(1):121-3

Millette B, Athanassoglou V, Patel A. High Flow nasal oxygen therapy in adult anaesthesia. Trends in Anaesthesia \& Critical Care 2018;18: 29-33. 